\title{
TOWARDS MORE EFFICIENT UAS DATA ACQUISITION: CAMERA AUTO MOUNT PIVOTING OBLIQUE SURVEY.
}

\author{
Igor Sales da Gama Campos \\ Eldorado Research Institute, igor.gama.90@gmail.com, Av. Mário Ypiranga, 315, Adrianópolis, Manaus, AM, 69057-070, Brazil
}

\author{
Commission I, II ICWG I/II
}

KEY WORDS: Aerial Surveying, Oblique Images, Remote Sensing, Photogrammetry, UAS, Flight Planning, Gimbaled Camera.

\begin{abstract}
:
In this paper I present a new MAVLink command, enabling oblique aerial surveys, along with its implementation on the major open source flight stacks (PX4 and ArduPilot) and ground control station (QGroundControl). A key advantage of this approach is that it enables vehicles with a typical gimbaled camera to capture oblique photos in the same pass as nadir photos, without the need for heavier and more expensive alternatives that feature multiple cameras, at fixed angles in a rigid mount, thus are unsuitable for lightweight platforms. It also allows for flexibility in the configuration of the camera angles. The principle is quite simple, the command combines camera triggering with mount actuation in a synchronized cycle along the flight traverses through the region of interest. Oblique photos have also been shown to increase the accuracy of data and help filling holes in point clouds and related outputs of surveys with vertical components. To provide evidence of its benefits, I compare the results of several missions, in simulated and field experiments, flown with nadir only surveys versus oblique surveys, and different camera configurations. In both cases, ground control and check points were used to evaluate the accuracy of the surveys. The field experiments show the vehicle had to fly $44 \%$ less with the oblique survey to cover the same area as the nadir survey, which could translate in a $80 \%$ gain in efficiency in coverage area per flight. Furthermore, this new command is capable of enhancing functionality of Unmanned Aerial Systems (UASs) without any additional hardware, therefore its adoption should be straightforward.
\end{abstract}

\section{INTRODUCTION}

The use of UASs as tools for data acquisition in photogrammetric surveys is becoming increasingly popular, likely due to the notable improvements on camera hardware, in contrast with the cost reduction of such systems as consequence of their mass commercialization as personal photography and film-making devices. Advancements in the technology benefit enterprise applications, which also leverage concepts from their military and manned counterparts. A recent example is the birth of UAS grade photogrammetric cameras featuring multiple sensors at nadir and oblique orientations, which range from industrial solutions (Leica Geosystems, 2017) to custom made rigs built with sensors found in mirrorless cameras (Foxtech, n.d.).

While the later oblique cameras are capable of being integrated into UASs with maximum takeoff weight below $25 \mathrm{~kg},{ }^{1}$ it certainly is not the case with the dominant, sub $2 \mathrm{~kg}$ vehicles, that are far more practical to operate as well as less expensive to acquire and maintain. This work focuses on enhancing the capabilities of these smaller systems, enabling them to perform surveys in broader scenarios while also being more efficient.

Several studies demonstrate the benefits of using oblique images in aerial surveys to increase accuracy (Nesbit and Hugenholtz, 2019) (Vacca et al., 2017) or fill data voids in areas otherwise ill observed (Li and Feng, 2018). Examples range from environments with greater degree of vertical components (Martínez-Carricondo et al., 2020), such as cities, construction (Varbla et al., 2020), canons, peaks, mountains (Sammartano et

\footnotetext{
1 The typical weight limit of simpler to obtain and operate licences. (Federal Aviation Administration, 2016) (Agência Nacional de Aviação Civil, 2017) (European Union Aviation Safety Agency, 2020) (Office of the Federal Register, 2020)
}

al., 2020) to other applications, such as power lines and utilities inspections (Jiang et al., 2017) (Wu et al., 2020), quarries and mines mapping (Rossi et al., 2017), glacier sheets erosion monitoring (Bates, 2020) and even forest inventory (Díaz et al., 2020). Moreover, the additional information from oblique images can also reduce errors (Nex and Remondino, 2014) and generate better 3D models (Lingua et al., 2017).

This work, however, focuses on another aspect that, despite being intrinsically related to nadir plus oblique surveys, to the best of my knowledge, has not been scientifically explored yet: the area covered by an UAS with oblique cameras can be considerably increased, thus allowing for a reduced number of flight traverses, which translates to higher efficiency. The rationale is that if one thinks of the images from the separate camera angles as a single output, the result would be a camera with much wider field of view, which means that the separation between flight lines can be bigger.

Instead of relying on cameras with multiple sensors placed in the same housing at fixed orientations (Xie et al., 2012) that end up being bulkier and heavier, the present approach uses a single camera that can be either integrated on a gimbal or mounted on a single servo adapter to allow actuation in the roll axis. The camera takes a picture at a first roll angle, the mount moves to the next angle and the process is repeated, when it reaches the final angle, it restarts from the beginning, repeating this cycle along the traverse. The rhythmical coordination of camera and mount is made seamlessly by the flight controller.

This approach became possible with advancements in camera technology, that allow continuously capturing photographs at faster rates, commonly allowing for few frames per second, in lightweight, compact systems. Because photogrammetric missions are performed in strong daylight and the exposure times 
are inherently low due to the motion of the vehicle, there are no special considerations regarding camera settings when compared to traditional, nadir surveys, as camera orientation is only changed after the exposure is complete. In fact, this approach might give sharper images, with less rotation induced blur, as gimbaled cameras are more stable.

The only foreseeable downside the author could speculate on is the inability to have a calibrated camera station, but it is likewise uncommon to have such care when using custom rigs made of consumer sensors, particularly as they might not be sufficiently rigid for maintaining accurate calibration stability. Notwithstanding, it is not an obstacle to employing direct georeferencing ${ }^{2}$ techniques, quite the contrary, it should excel in this scenario, as the orientation information would likely be more valuable with oblique images than nadir ones.

Summarizing, this new oblique survey technique enables smaller UASs to capture data in the same fashion as their heavier counterparts featuring dedicated, more expensive oblique camera rigs. This could ultimately be used to boost efficiency or increase the accuracy in specific scenarios, particularly the ones involving vertical features and facades.

\section{MATERIALS AND METHODS}

The proposed approach, Camera Auto Mount Pivoting Oblique Survey (CAMPOS), relies on a newly created MAVLink (MAVLink Protocol Maintainers, 2020b) command to coordinate the acquisition of images along a flight traverse through the region of interest. However, this work is not limited to the specification of this new command, but also entails its implementation in PX4 (PX4 Development Team, 2020) and ArduPilot (ArduPilot Development Team, 2020) flight stacks and QGroundControl (QGroundControl Development Team, 2020) Ground Control Station (GCS). By implementing in these autopilots I enable wide adoption and integration as they are the major free and open source software alternatives, while QGroundControl guarantees a wide coverage of devices through the same code, as it is implemented in Qt, allowing the simultaneous release of $\mathrm{PC}$ and mobile software for multiple platforms.

It shares the same concept as the S.O.D.A. 3D by senseFly (senseFly, 2016), while not being limited to a specific hardware or platform. Whereas my changes in QGroundControl also account for the wider field of view and number of photo orientations while computing frontlap and sidelap, but senseFly suggests different settings for these variables, making it appear that their GCS disregards the effect of the oblique survey settings on them. Moreover, the present approach leans more on increased coverage per flight, in contrast to senseFly's, which concentrates on higher accuracy in specific scenarios.

\subsection{MAVLink Command}

The newly created MAVLink command (Campos, 2020c) behaves similarly to a preexisting command that instructs the flight controller to capture images by distance. The new functionality is the definition of positions that the camera should take pictures through the flight traverse by the specification of the following parameters:

2 Direct georeferencing refers to determining the position and orientation of each photograph (Mostafa et al., 1997), which is typically achieved by having a geodetic grade, multiple frequencies GNSS receiver with RTK/PPK solutions onboard the vehicle and a high performance IMU attached to the camera to provide high accuracy position and attitude information for the captured images. (Eling et al., 2014)

\subsubsection{MAV_CMD_OBLIQUE_SURVEY parameters.}

1. Distance: camera trigger distance (in meters)

2. Shutter: camera shutter integration time (in milliseconds)

3. Min Interval: The minimum interval in which the camera is capable of taking subsequent pictures repeatedly. (in milliseconds)

4. Positions: Total number of roll positions at which the camera will capture photos (images captures spread evenly across the limits defined by Roll Angle) (integer)

5. Roll Angle: Angle limits that the camera can be rolled to left and right of center (in degrees)

6. Pitch Angle: Fixed pitch angle that the camera will hold in oblique mode if the mount is actuated in the pitch axis (in degrees)

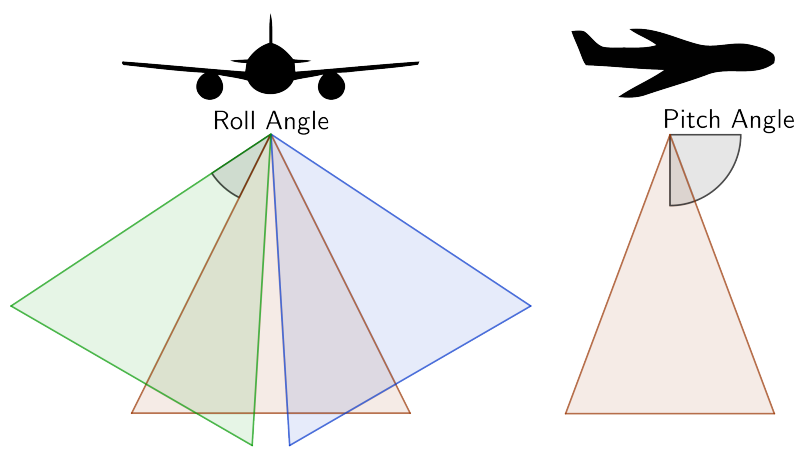

Figure 1. CAMPOS command parameters.

To better illustrate how these parameters specify the camera orientations one can refer to Figure 1, where the angles highlighted in black accompany their labels. In the given example the number of Positions is 3, hence, from the frontal perspective there are three different image captures, one of which is a nadir image, as the angles defined by Roll Angle are evenly spread according to the number of positions and Pitch Angle is $-90^{\circ}\left(0^{\circ}\right.$ is facing forward). Conversely, if the parameters specified for position, roll and pitch angles are respectively $3,20^{\circ}$ and $-90^{\circ}$, the autopilot will take a picture at roll angles $-20^{\circ}, 0^{\circ}$ and $20^{\circ}$, then revert back to $-20^{\circ}$ and repeat this cycle, changing orientation every time it triggers the camera until a new CAMPOS command orders its stop through Distance of $0 \mathrm{~m}$. Shutter is to help the autopilot know how long it needs to keep the camera shutter pressed, whereas Min Interval is the amount of time the autopilot will wait for the camera to capture the image before altering the mount's roll angle.

\subsection{Autopilot Logic}

To implement the CAMPOS command (Campos, 2020a) (Campos, 2020b) I slightly changed the logic to trigger the camera by distance, adding the necessary instructions to move the camera mount when the right time is reached.

Since it is uncommon and unnecessary to have feedback from the mount actuators, there is no verification whether the mount reached the target position before the camera is triggered, therefore, when planning the mission, the operator must ensure that the camera triggering and actuation rate is achievable, as these are system specific variables, with proper values there will be no motion during camera exposure. Generally, what mostly influences the maximum rate is the camera continuous shooting capability, as servos typically operate at approximately $600 \%$, 
and should therefore take approximately $0.15 \mathrm{~s}$ to actuate from one extreme to the other, if a highly oblique roll angle of $45^{\circ}$ is used. This design decision ensures compatibility with a greater number of platforms.

My changes in the source code do not alter the behavior of the photo time-stamping or the information logged in the camera triggering messages, which presently hold the position and orientation of the vehicle rather than the camera, in both PX4 and ArduPilot. Unfortunately this is a limiting factor for the use of direct georeferencing, and should be of consideration for future work by both firmware teams.

Figure 2 provides a conceptual flowchart of the CAMPOS logic in both autopilots stack, as the principle is essentially the same, differing only in the actual code to adapt to each of the flight stacks' existing architecture.

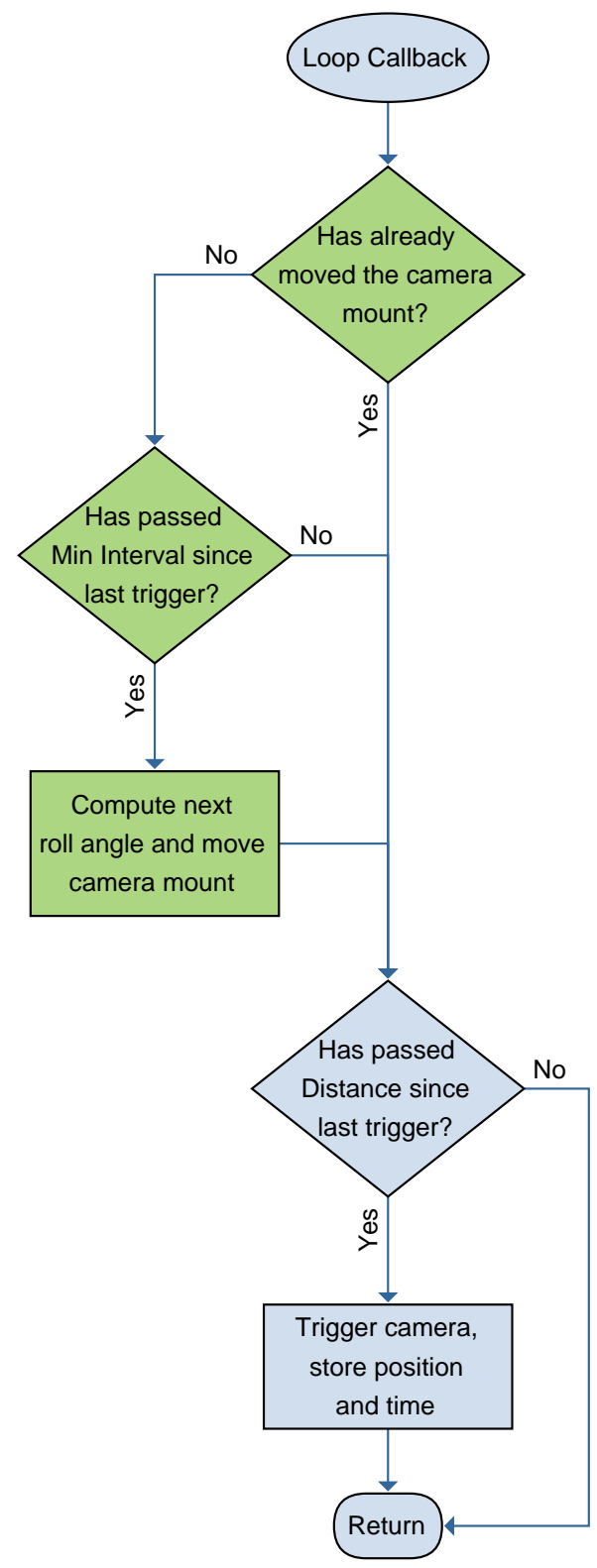

Figure 2. CAMPOS autopilot logic additions in green.

\subsection{QGroundControl Changes}

The bulk of the calculations to channel the increased horizontal field of view from the new command into efficiency gain is done in the GCS. As previously mentioned, QGroundControl was chosen due to its ubiquitous availability in several platforms, from PCs to mobile devices, while being OS agnostic thanks to the Qt framework. Furthermore, it is already an industry standard and many UAS manufacturers use it directly or through a custom-flavored skin. (MAVLink Protocol Maintainers, 2020a)

To allow seamless integration in QGroundControl's source code, the implementation of CAMPOS method (Campos, 2020d) computes new values for the projected image footprint on the ground, then leaves for the existing code to do the path planning and waypoint generation. To achieve the optimal configuration, we adjust the height of the survey with respect to the target Ground Sampling Distance (GSD), camera intrinsics, roll angle and sidelap settings, ensuring the desired GSD will be achieved through the centermost, core part of the virtual image that will be used in orthomosaic outputs.

We generalize the projected image width as if the Angle of View (AoV) was the camera's one plus twice the configured roll angle, upper bounded to $150^{\circ}$ to limit the footprint size during the calculation, what I refer from hereon as virtual image. This limits degradation in the image resolution induced from a virtual AoV that is too wide, as above $90^{\circ}$ the gain of information from increasing the AoV decreases inversely proportionally to the tangent of half the AoV, but since what determines the survey's GSD is the centermost part of the image, it would not be sensible to limit the virtual AoV to merely $90^{\circ}$.

One might note that the photographs defined by the target number of positions will be displaced by the triggering distance, as the vehicle will continue flying at a constant speed through the process, yet it is not unreasonable to assume otherwise and compute the AoV in this manner as the captured images will follow the same diagonal pattern along every flight traverse. Additionally, for the vertical footprint adjustment, we simply divide the distance by the number of positions, guaranteeing that the target frontlap will be achieved by every subsequent image of same orientation, which should be noted by the operator, as a smaller value might be sufficient for the mission.

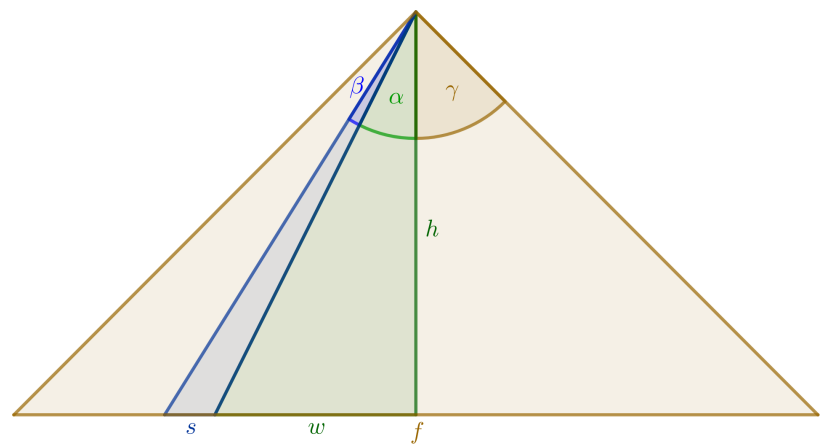

Figure 3. GSD illustration.

To achieve the desired effect, the computations are made with regard to the GSD on a pixel basis, instead of the average of the image, similar to what is done by (Lingua et al., 2017). That makes it possible to calculate the optimum height to achieve the intended GSD. Figure 3 shows the triangles that provide the relation between height, GSD, camera intrinsics, sidelap and roll angle settings. 
From the trigonometric relations we get the following initial equations:

$$
\begin{aligned}
\frac{s+w}{h} & =\tan (\alpha+\beta), \\
\frac{w}{h} & =\tan (\alpha), \\
f & =2 \tan (\gamma) h \\
(1-o) f & =2 \tan (\alpha) h,
\end{aligned}
$$

which, by plugging 2 into 1 and 3 into 4 , result in the consecutive relations:

$$
\begin{aligned}
\alpha & =\tan ^{-1}((1-o) \tan (\gamma)) \quad \text { and } \\
h & =\frac{s}{\tan (\alpha+\beta)-\tan (\alpha)},
\end{aligned}
$$

finally leading to:

$$
h=\frac{s}{\tan \left(\tan ^{-1}((1-o) \tan (\gamma))+\beta\right)-(1-o) \tan (\gamma)},
$$

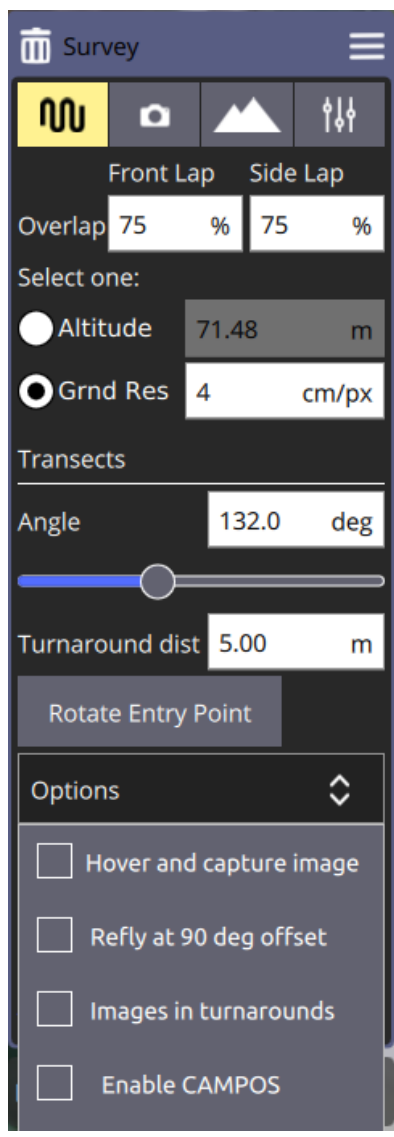

(a) CAMPOS toggle

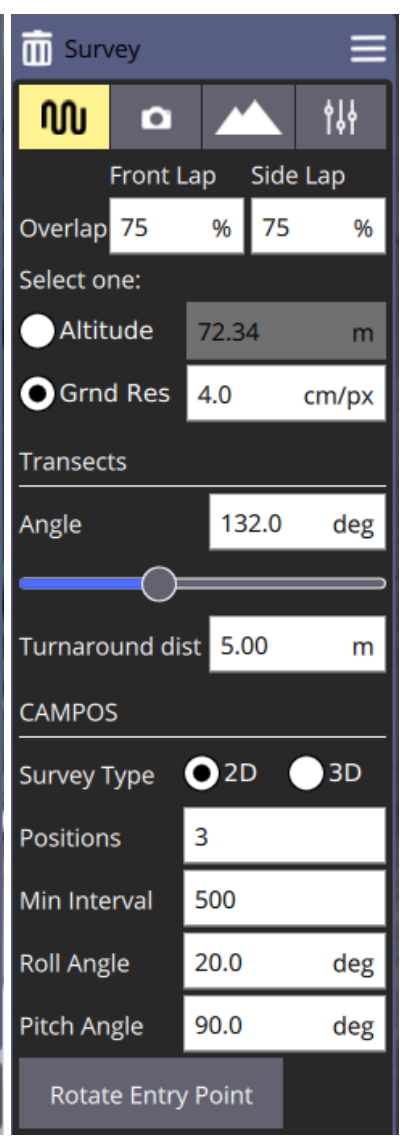

(b) CAMPOS enabled
Figure 4. CAMPOS user interface in QGroundControl.

$$
\text { where } \begin{aligned}
h= & \text { height } \\
s= & \text { GSD } \\
o= & \text { ratio of sidelap } \\
w= & \text { half the width of the core portion from the virtual } \\
& \text { image } \\
\alpha= & \text { half the AoV of the core part from the virtual im- } \\
& \text { age } \\
f= & \text { the field of view from the virtual image } \\
\gamma= & \text { half the angle of view from the virtual image } \\
\beta= & \text { the angular pitch of the pixel (exaggerated view) }
\end{aligned}
$$

To have the least impact on user interaction with QGroundControl's interface, the CAMPOS method was added as a toggle option inside the survey menu, if checked, its respective parameters are shown. Figure 4 shows the two states. The pitch angle has the opposite sign in the user interface to keep consistency with the other commands that control camera pitch in QGroundControl and other GCSs. The shutter parameter is omitted as it is not currently implemented in the autopilots, but as it shares the same concept as the camera trigger by distance, when implemented, it should work seamlessly for both. In this example, it is also possible to see that the height from a nadir survey differs from the CAMPOS' one, this is consequence of the computation from Equation 7. I later added a toggle to enable or disable this computation (2D or 3D survey type), allowing for use of the CAMPOS method with the same traverses as a regular survey, albeit capturing multiple angles in a single flight.

\subsection{Simulated Test Bed}

To ensure employing the CAMPOS method would not adversely affect the surveys, I performed tests in a simulated environment with Gazebo and PX4's Software In The Loop (SITL) simulator running the Typhoon H480 model in Sonoma Raceway world. This world was chosen as it was generated from a laser scan of the actual location, thus providing a realistic scenario for the simulation. The downside, however, is that the textures of the model are repeating, generated patterns instead of actual photographs, which makes it harder for the photogrammetry processing software to find matches, causing lower accuracy than real scenarios, this also led to a hole with both CAMPOS and the traditional surveying methods in an area where the camera would only capture grass patterns.

To mimic a real-world survey, I added several virtual Ground Control Points (GCPs) to the simulated world, with no geolocation error and updated the camera parameters to match the Sony RX0's resolution and field of view, which features an 1" 20 MP sensor with $2.4 \mu \mathrm{m}$ pixel pitch, just like the Phantom 4 Pro, an established UAS for photogrammetric applications, albeit in RX0 the image is cropped to a $15.36 \mathrm{MP}, 4800 \times 3200$ output.

\subsection{Field Experiments Test Bed}

After the simulated tests shown that the accuracy of the CAMPOS method and the nadir survey were similar, I decided to perform field experiments to finally eliminate any other factor that could adversely affect the quality of the survey. I followed the same protocol as with the simulated experiments, although on a smaller scale. I staked 16 GCP positions of which 5 were used as check points and flew over a $0.05 \mathrm{~km}^{2}$ area comprising five blocks of houses, trees, parking lots and a couple buildings with three or four stories.

The UAS used for flight testing the method is a 3DR Solo, the reason is that it features the open-source flight stack ArduCopter, the multicopter flavor of ArduPilot. It features the 
original flight controller and camera gimbal, nonetheless, updated to the OpenSolo 4 software, featuring only the CAMPOS' changes that were cherry-picked from the development branch to the stable 4.0.5 version of ArduCopter, specifically compiled for the CubeSolo. In the process I stumbled upon a bug which caused the gimbal to ignore roll angle inputs and promptly provided a fix for it.

The camera used was the GoPro Hero 3+ Black, that, albeit old, is one of the few options compatible with Solo's gimbal, the only newer being the GoPro Hero 4, which features a sensor of the same size and resolution (12 MP), varying only slightly the focal length. Since this camera has a fisheye lens, which is not a popular type for aerial surveying applications due to the low GSD inherent from the design, I set it up to capture 7 MP photos in a narrower field of view with $3000 \times 2250$ pixels, which according to (GoPro, 2019) results in a $94.4^{\circ}$ horizontal AoV. This choice makes it better represent the typical scenarios for aerial surveys.

To stake the GCPs a ComNav AT330 geodetic, multi-frequency (L1, L2 and L5), multi-constellation (GPS, Glonass, Galileo and Beidou) antenna attached to a $2 \mathrm{~m}$ carbon fiber rod was used in conjunction with a ComNav K706 OEM board (L1/L2, GPS, Glonass, Galileo) as well as with an u-blox NEO-M8T (L1, GPS, Glonass, Galileo). In both cases a stationary observation station approximately $2.5 \mathrm{~km}$ away from the Brazilian continuous monitoring network featuring a Trimble NetR9 receiver paired with a Zephyr 2 geodetic antenna was used as a base station. To ensure accurate data, the survey was performed in stop-and-go fashion with observation time of 5 minutes in each point and the raw observation files were post-processed with bidirectional corrections with (Everett, 2020). The location of each point was determined by the centroid of the fixed solutions within the corresponding observation interval.

\section{SIMULATED EXPERIMENTS}

To validate the effectiveness of CAMPOS method I ran a survey mission on the simulated environment described in subsection 2.4. The mission was configured for a $5 \mathrm{~cm} / \mathrm{pix}$ GSD with both techniques. For the nadir survey, $80 \%$ frontlap and $70 \%$ sidelap were used, while for the CAMPOS one, the same frontlap and a slightly larger $75 \%$ sidelap were used, the additional parameters were 3 positions, $20^{\circ}$ roll angle and $90^{\circ}$ pitch. I wanted to show that even with higher sidelap settings the CAMPOS method would use far fewer traverses, while for nadir I used a common choice of overlap settings.

Figure 5 shows the location of photos for each method. To cover the same area CAMPOS used 9 traverses while nadir used 16 .

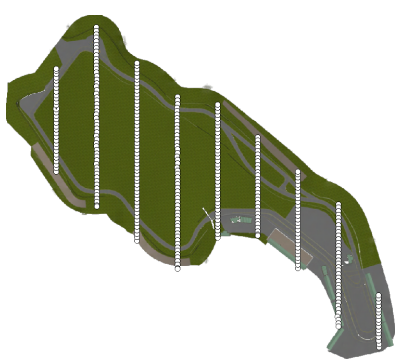

(a) CAMPOS

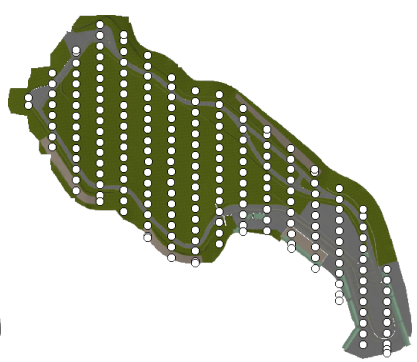

(b) nadir
Figure 5. Location of photos with each survey technique.
The flight length was 9,283 $\mathrm{m}$ for the nadir survey and 5,994 $\mathrm{m}$ for the CAMPOS one, which translates in an approximately $35 \%$ smaller fight, and, conversely, approximately $55 \%$ higher efficiency. Despite that, as CAMPOS guarantees that every subsequent image in the same orientation will have the specified frontlap setting, it produced 338 photos, whereas nadir 213.

To mark the GCPs I created a model of $1 \mathrm{~m} \times 1 \mathrm{~m}$ chessboard in a $2 \times 2$ grid, then added it to several places in the original sonoma raceway model. A total of 81 points were used and their locations was determined through an ideal GNSS sensor at its center. The GCPs indexes start at zero and go all the way up to 80. Even numbers were used as control points and odd numbers as check points. Their locations is shown in Figure 6

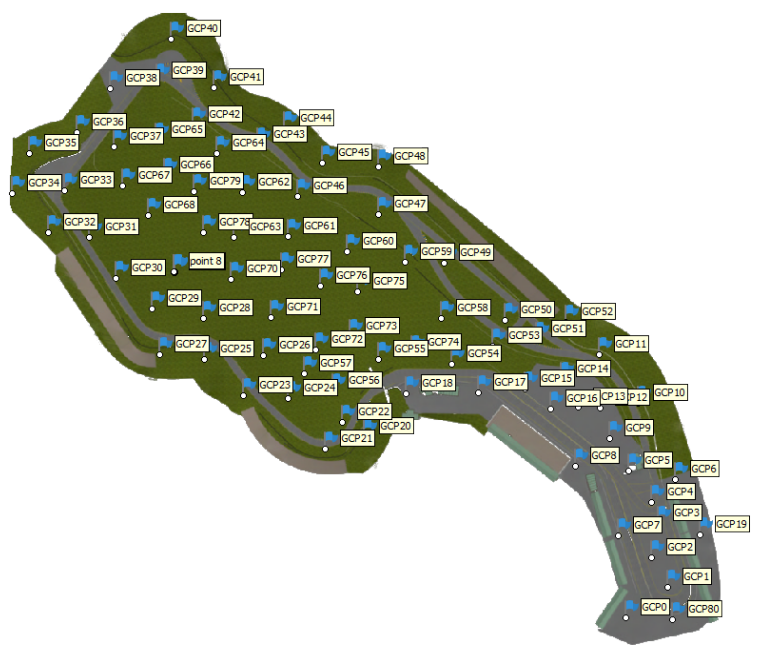

Figure 6. GCPs locations on the Sonoma Raceway virtual world.

The results from processing the surveys in Agisoft Metashape (Agisoft, 2020) are displayed in Table 1. The settings were for high accuracy in both, alignment and dense cloud generation and aggressive filtering for the later. Both methods failed to align three images each and ended up with a hole in the dense cloud. Figure 7 also show a smaller overlap, particularly notable on the nadir survey due to the higher terrain elevation in that region, as the UAS took off from the lower part of the terrain, to the southeast of the surveyed area.

\begin{tabular}{l|c|c|c|c}
\multicolumn{1}{c|}{ Method } & Type & XY error & $\mathbf{Z}$ error & Total \\
\hline \multirow{2}{*}{ CAMPOS } & Control & 0.384 & 0.109 & 0.399 \\
& Check & 0.924 & 0.453 & 1.030 \\
\hline \multirow{2}{*}{ nadir } & Control & 0.486 & 0.116 & 0.499 \\
& Check & 1.370 & 0.826 & 1.600
\end{tabular}

Table 1. GCPs root mean square error, units in meters.

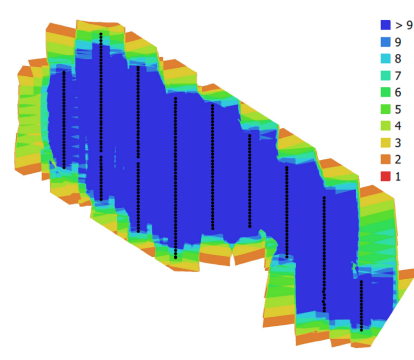

(a) CAMPOS

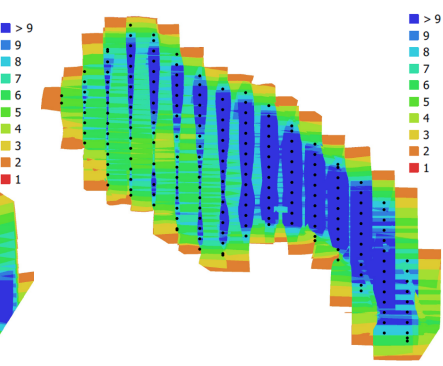

(b) nadir
Figure 7. Overlap of photos with each survey technique. 
As this was a simulated environment, it was possible to compare the dense clouds generated from the surveys with the original model supplied to the simulator. I used CloudCompare (CloudCompare Development Team, 2020), a free and open source $3 \mathrm{D}$ point cloud and mesh processing software to evaluate these differences and also compared the two dense-clouds with each other. To align the point clouds with the model, I manually matched them to the best of my ability and finely registered them using the model as reference through the software's automated process.

Figure 8 shows the distances between the source model and the dense point clouds generated from each survey method. The black areas are holes due to lack of features in the region, as it consisted solely of grass, it is, however, smaller in the CAMPOS survey due to the oblique images around the area that successfully aligned and provide more information about the area. The nadir survey also failed to align a few parts at the borders of the region, albeit having overhead imagery of them, this is more pronounced in the left side of the surveyed area. Despite these differences, it is notable the striking similarity between each distance map, showing the CAMPOS method is a valuable surveying tool.

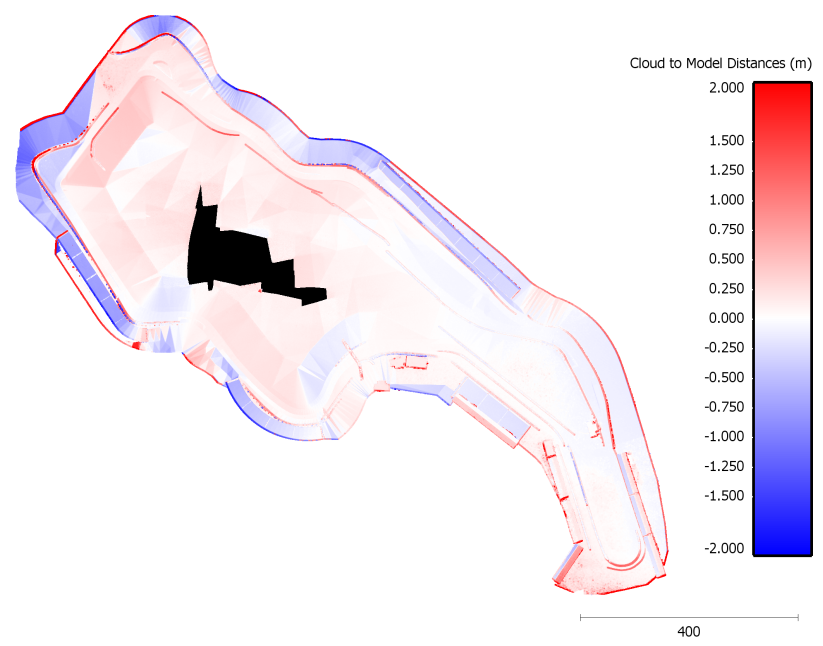

(a) CAMPOS

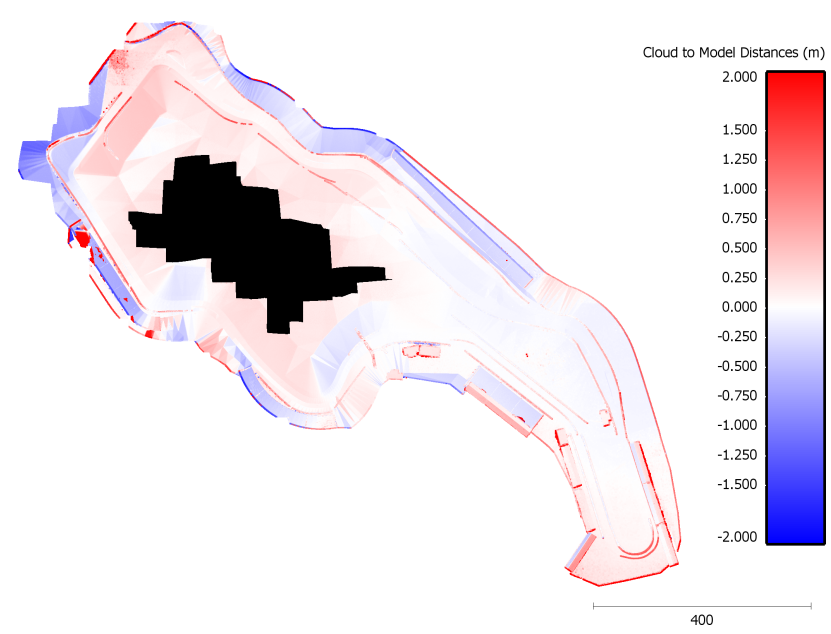

(b) nadir

Figure 8. Distances between the original model and the dense point clouds generated from each survey method. The black areas show a hole in alignment that occurred due to low texture.

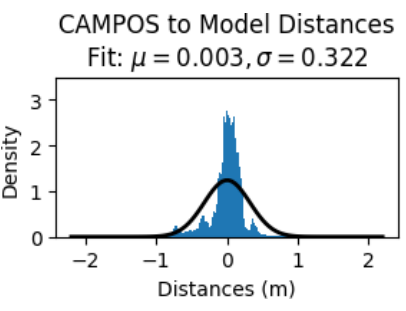

(a) CAMPOS

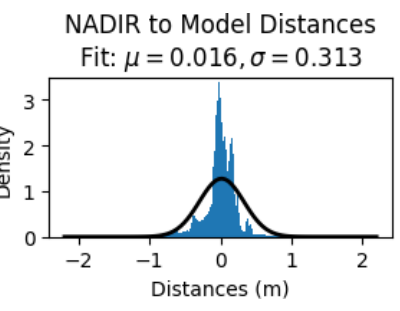

(b) nadir
Figure 9. Density histograms along the Gaussian distribution fits of distances between the original model and the dense point clouds generated from each survey method.

Figure 9 shows the histograms of the distances between the source model and the dense point clouds along the Gaussian distribution fit for each survey method. The CAMPOS method achieved higher accuracy, with a mean deviation of $0.003 \mathrm{~m}$, while nadir reached $0.016 \mathrm{~m}$, on the other hand, nadir was more precise and achieved $0.313 \mathrm{~m}$ standard deviation, while CAMPOS reached $0.322 \mathrm{~m}$. Nonetheless, these numbers are extremely close, especially if one considers the target GSD was $5 \mathrm{~cm} /$ pix and the variations are in the order of $1 \mathrm{~cm}$.

To finalize the comparison of the simulated experiments between CAMPOS and nadir, I computed the distances between each point cloud. To eliminate the difference in the voids, the maximum distance was limited to $0.2 \mathrm{~m}$ after determining that more than $95 \%$ of the values lied below it. This allowed us to see how consistent the reconstruction was with the different survey methods. Figure 10 shows the absolute distances between the dense point clouds. A histogram is present at the right of the legend and it is clear the majority of values (58\%) is below $0.05 \mathrm{~m}$, which was the target GSD.

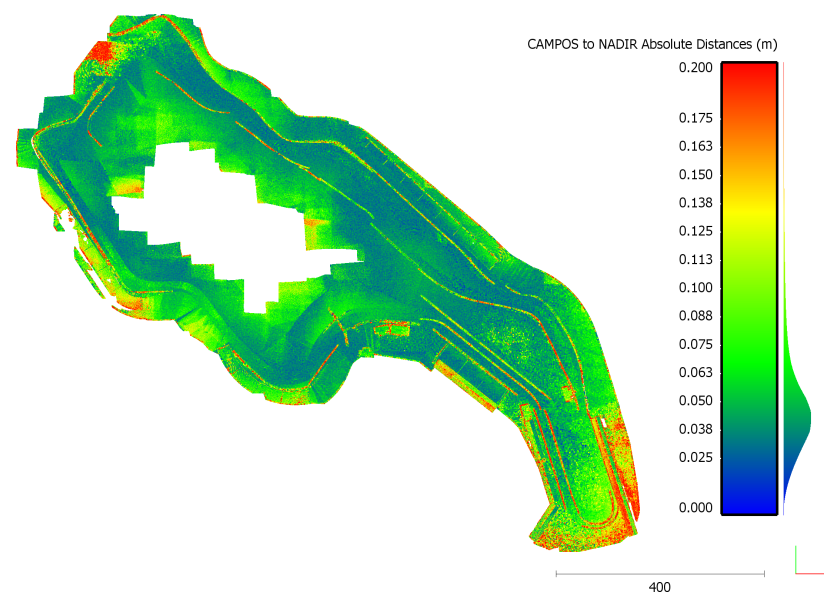

Figure 10. Distances between CAMPOS and nadir dense point clouds.

\section{FIELD EXPERIMENT}

To ultimately validate the feasibility of using the CAMPOS method as a way to enhance surveying efficiency without adversely affecting the accuracy of the outputs, I performed field experiments with accurately staked GCPs. Differently from the simulated environment, there is no accurate 3D model of the area to be used as a ground-truth, but the photogrammetry software report should be sufficient to demonstrate the equivalent accuracy of either method. 


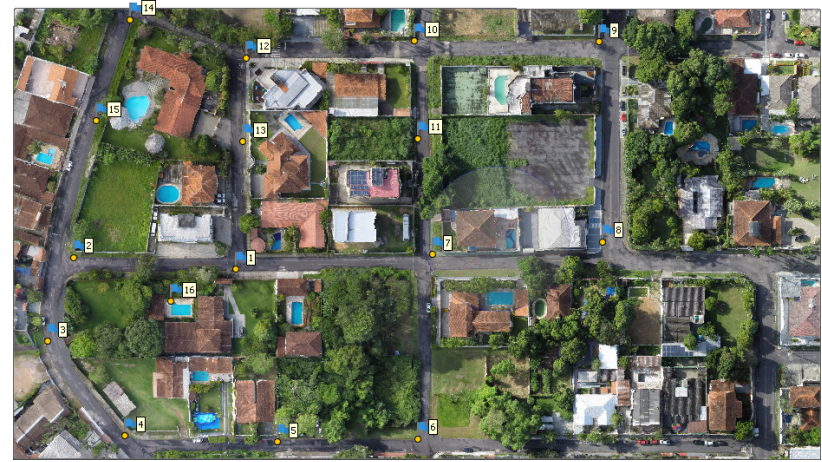

Figure 11. GCPs locations of field experiments.

A total of 16 GCPs were measured and marked with white paint on the asphalt, in a $50 \times 50 \mathrm{~cm}, 2 \times 2$ grid, chessboard pattern, with the exception of one, which is at the corner of a swimming pool on the site used as operation area, labeled as 16 . Of these points, 5 were used as check points, they are numbered 4, 7, 10, 13 and 16. The GCPs locations are shown in Figure 11.

The survey was set to $4 \mathrm{~cm} /$ pix GSD and $75 \%$ frontlap and sidelap for the nadir method and $60 \%$ frontlap, $75 \%$ sidelap for the CAMPOS method. The area consisted of part of a residential neighbourhood comprising five blocks. It was processed for high accuracy in alignment and dense cloud generation, with moderate filtering for the later. According to Metashape's reports both methods achieved $3.88 \mathrm{~cm} /$ pix GSD.

Figure 12 show the overlap of photos for each method as well as their positions. In this scenario CAMPOS used 5 flight traverses while nadir resulted in 9 flight traverses. Another key aspect is that the nadir survey had substantially less overlap on the edges of the area, which was intensified on the corners, this effect was only mildly observed at the corners with the CAMPOS method.

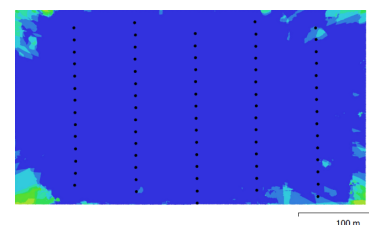

(a) CAMPOS

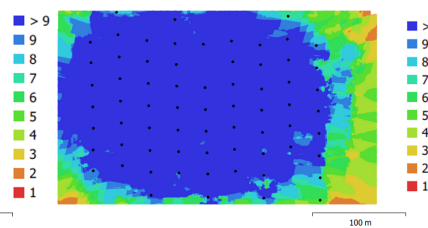

(b) nadir
Figure 12. Locations and overlap of photos with each method.

Table 2 shows the resulting errors for the processed surveys with both methods in Agisoft Metashape, which are in line with the general rule of thumb that states the expected accuracy is around two to three times the GSD, they are a bit less accurate, but this could be explained by the poor optics of the GoPro camera and the increased distortion inherent to the fisheye lens design. Most importantly, both methods achieved quite similar accuracies, varying only a few millimeters, which, being less than half the GSD could well mean these differences are due to slight offsets while manually marking the GCPs on the images or even numerical variations in the processing of the data.

\begin{tabular}{l|c|c|c|c}
\multicolumn{1}{c|}{ Method } & Type & XY error & Z error & Total \\
\hline \multirow{2}{*}{ CAMPOS } & Control & 9.21 & 3.93 & 10.01 \\
& Check & 15.02 & 3.02 & 15.32 \\
\hline \multirow{2}{*}{ nadir } & Control & 9.16 & 3.30 & 9.74 \\
& Check & 14.13 & 4.20 & 14.74
\end{tabular}

Table 2. GCPs root mean square error, units in centimeters.

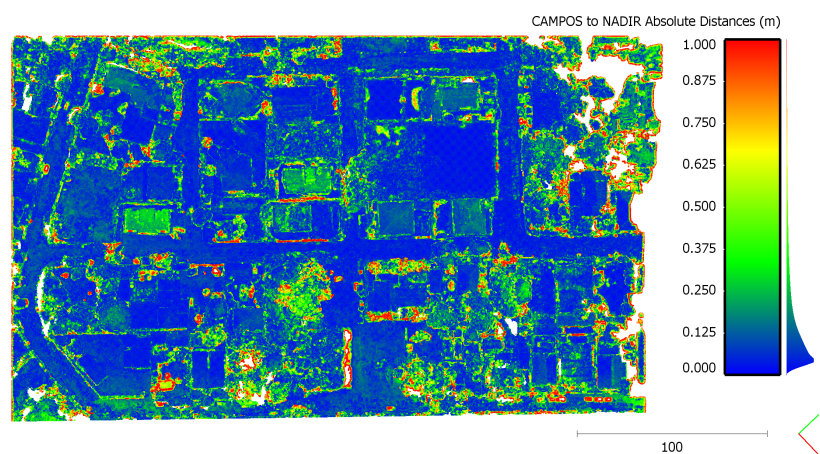

Figure 13. Distances between CAMPOS and nadir dense point clouds.

Figure 13 highlights the distances between the dense point clouds produced with each method. Since the scene features dynamic content, such as parked cars present in one, but not the other and trees subject to wind and changes in foliage, the differences are more pronounced than in the static, simulated environment. I limited the distances to $1 \mathrm{~m}$ for better visualization on the comparison map, almost $85 \%$ of the non filtered distances lie below it, while nearly $76 \%$ are below $0.5 \mathrm{~m}$ and approximately $50 \%$ are within $0.15 \mathrm{~m}$ away. It is interesting to note the distances are slightly larger at the rooftops, but particularly accentuated on the top of the three and four stories buildings (respectively at the center and mid-left portion of the image), however, I believe the reason for that is the lack of textures in the materials of these roofs, which generated poorer reconstruction, the distances are not observed on the same degree at the rooftop of a house at higher ground to the mid-right, despite being at the same distance to camera as these buildings.

\section{FUTURE WORK}

Since the focus of this work is towards increased efficiency of photogrammetric UAS surveys for the traditionally $2 \mathrm{D}$ outputs of orthomosaic and digital elevation models through oblique imagery, it remains to be done in future work the evaluation of employing the CAMPOS method for the generation of 3D models, as it has the potential to present even higher efficiency gains for those applications, because they typically require the oblique images, which are presently captured in many flights.

As an example, if one intends to make an aerial survey to create a 3D model of a neighbourhood or similar larger scale project, today's alternative consist of flying the UAS with fixed pitch in a double grid pattern, but to acquire images facing the same direction with a given sidelap, its non-overlapping ratio must be halved, as neighbouring traverses have opposite directions. This must be done for each target oblique angle, plus a nadir survey, which translates in a minimum five fold increase in flight length for such surveys when compared to typical nadir only surveys, potentially more if multiple oblique angles are desired.

Using the CAMPOS method without employing the mechanism to adjust the horizontal footprint of the images, the UAS can capture the same data in a simple double grid mission, with no increase in sidelap ratio, which would translate in a twofold only increase in flight length when compared with a typical nadir only survey. Moreover, CAMPOS is capable of capturing two or more oblique angles with the same flight length, perhaps 
needing adjustments to the vehicle's speed to match the camera's continuous shooting capabilities.

Through an extension of the CAMPOS method allowing varying of both pitch and roll, it would be possible to perform this kind of mission in a single lawnmower pattern flight, however, it would decrease vehicle compatibility, as it would require a two axis gimbaled camera, increasing complexity and reducing commonality between differently capable platforms, such as roll only gimbaled fixed-wing aircraft versus 3D gimbaled rotorcraft. Further consideration would also be needed regarding the overlap with respect to the camera's aspect ratio.

Considering this goes beyond the scope of this work, I hope to explore these possibilities in future research, while remaining cautiously excited from these initial indications.

\section{CONCLUSION}

In this work I propose, implement and evaluate the performance of a novel UAS surveying method focused on increasing the efficiency of vehicles capable of actuating the camera mount in the roll axis. It consists of the definition of a new MAVLink command to support the underlying autopilot logic that seamlessly orchestrates the camera triggering and mount actuation to enable capturing of nadir and multiple oblique images in a single flight traverse. To leverage the full potential of the concept, a new approach to compute the side overlap of images had to be devised and integrated into the GCS. I named this triad of contributions the CAMPOS method, after Camera Auto Mount Pivoting Oblique Survey.

Through extensive and careful analysis of simulated and field experimentation I demonstrated the technique is capable of increasing the efficiency of aerial surveying, while producing equivalent output to a nadir only survey. In fact, after extrapolating the flight plans to a bigger area to calculate the efficiency gain when comparing the two methods, a $6.22 \mathrm{~km}^{2}$ area would be mapped in approximately $105 \mathrm{~km}$ with CAMPOS and 213 $\mathrm{km}$ with a nadir only survey, using a $20^{\circ}$ roll angle and $75 \%$ for both frontal and side overlap, this would translate in over $50 \%$ savings and $100 \%$ higher efficiency. If the roll angle was $30^{\circ}$, the flight length would be approximately $82 \mathrm{~km}$, raising the gains to $62 \%$ savings and $160 \%$ efficiency. For choosing the angles, I relied on (Nesbit and Hugenholtz, 2019). Flight lengths and number of traverses vary with different cameras, but the ratios of gains remain practically unchanged for cameras with up to $80^{\circ}$ of $\mathrm{AoV}$.

However impressive these numbers look, as they have not been experimentally verified, I cautiously claim the gains observed in the field experiment, where the sidelap setting for both cameras were the same (44\% savings for the same area or, conversely, $80 \%$ increased efficiency). One can note in Figure 12 that the nadir flight plan resulted in sub-optimal coverage and would in fact benefit from another traverse at the right side, which would coincide with the findings of the aforementioned estimates.

The CAMPOS method is a clever and effective way of utilizing existing hardware in new ways to enhance efficiency, it is also already implemented in the leading free and open source flight stacks and GCS, requiring only minimal camera actuation capabilities, therefore I am confident that it will have a smooth transition to widespread adoption by UAS manufacturers.

\section{ACKNOWLEDGEMENTS}

First, I would like to thank the Eldorado Research Institute for funding my participation in the XXIV ISPRS Congress, enabling the publication of this work. Furthermore, the discussions with the members and contributors of the open source projects MAVLink, PX4, ArduPilot and QGroungControl were paramount in transforming what was initially a concept and suggestion into a new feature with implementation in all of these projects and making me see it through to the end. I especially thank Mr. Don Gagne and Mr. Hamish Willee for their opinions and suggestions.

\section{REFERENCES}

Agência Nacional de Aviação Civil, 2017. Requisitos Gerais para Aeronaves Não Tripuladas de Uso Civil - RBAC-E n ${ }^{\circ} 94$. http://www.anac.gov.br/assuntos/legislacao/legislacao-1/rbhae-rbac/rbac/rbac-e-94-emd-00 (7 January 2021).

Agisoft, 2020. Agisoft Metashape Photogrammetry Software. https://www.agisoft.com/ (9 January 2021).

ArduPilot Development Team, 2020. Open Source Drone Software. ArduPilot. Software. https://ardupilot.org/ (7 January 2021).

Bates, J. S., 2020. Oblique UAS Imagery and Point Cloud Processing for 3D Rock Glacier Monitoring. Master's thesis, University of Münster.

Campos, I. S. G., 2020a. add Camera Auto Mount Pivoting Oblique Survey (CAMPOS). Github. https://github.com/PX4/P X4-Autopilot/pull/15882 (7 January 2021).

Campos, I. S. G., 2020b. add CMD260: Camera Auto Mount Pivoting Oblique Survey (CAMPOS). Github. https://github.c om/ArduPilot/ardupilot/pull/15878 (7 January 2021).

Campos, I. S. G., 2020c. add command 260 for Oblique Survey. Github. https://github.com/mavlink/mavlink/pull/1508 (7 January 2021).

Campos, I. S. G., 2020d. add pseudo oblique function to CMD 206 CAM_TRIGG_DIST. Github. https://github.com/mavlink /qgroundcontrol/pull/9103 (7 January 2021).

CloudCompare Development Team, 2020. CloudCompare Software. https://www.danielgm.net/cc/ (9 January 2021).

Díaz, G. M., Mohr-Bell, D., Garrett, M., Muñoz, L., Lencinas, J. D., 2020. Customizing unmanned aircraft systems to reduce forest inventory costs: can oblique images substantially improve the 3D reconstruction of the canopy? International Journal of Remote Sensing, 41(9), 3480-3510. https: //doi.org/10.1080/01431161.2019.1706200.

Eling, C., Klingbeil, L., Kuhlmann, H., 2014. A Precise Direct Georeferencing System for UAVs. Proceedings of the Workshop on UAV-basaed Remote Sensing Methods for Monitoring Vegetation, 94, 33-41. https://doi.org/10.5880/TR32DB.KGA94.6.

European Union Aviation Safety Agency, 2020. Open Category - Civil Drones. EASA. https://www.easa.europa.eu/domains/c ivil-drones-rpas/open-category-civil-drones (7 January 2021).

Everett, T., 2020. Customized RTKLIB Software, Version Demo5 b33e. rtklibexplorer.com (9 January 2021). 
Federal Aviation Administration, 2016. SUMMARY OF SMALL UNMANNED AIRCRAFT RULE (PART 107). FAA. http://www.faa.gov/uas/media/Part_107_Summary.pdf (7 January 2021).

Foxtech, n.d. Foxtech 3DM V3 3D photogrammetry Mapping Survey Oblique camera. Foxtech. https://www.foxtechfpv.com /foxtech-3dm-v3-oblique-camera-for-mapping-and-survey.h tml (7 January 2021).

GoPro, 2019. GoPro HERO3+ Black Edition Field of View (FOV) Information. https://community.gopro.com/t5/en/HE RO3-Black-Edition-Field- of-View-FOV-Information/tap/390085 (9 January 2021).

Jiang, S., Jiang, W., Huang, W., Yang, L., 2017. UAV-Based Oblique Photogrammetry for Outdoor Data Acquisition and Offsite Visual Inspection of Transmission Line. Remote Sensing, 9(3), 278. https://doi.org/10.3390/rs9030278.

Leica Geosystems, 2017. Leica RCD30 for UAV. Leica Geosystems. https://leica-geosystems.com/-/media/files/leicageos ystems/products/datasheets/leica_rcd30_for_uav_ds_en.ashx?1 $\mathrm{a}=$ fr- be\&hash=F93FFF9B9C48D6E2547A41048C3EA934 (7 January 2021).

Li, S., Feng, L., 2018. Establishment of High Precision Terrain Model of Eroded Gully with UAV Oblique Aerial Photos. IOP Conference Series: Materials Science and Engineering, 452, 022027. https://doi.org/10.1088/1757-899X/452/2/022027.

Lingua, A., Noardo, F., Spanò, A., Sanna, S., Matrone, F., 2017. 3D MODEL GENERATION USING OBLIQUE IMAGES ACQUIRED BY UAV. ISPRS - International Archives of the Photogrammetry, Remote Sensing and Spatial Information Sciences, XLII-4/W2(4W2), 107-115. https://doi.org/10.5 194/isprs-archives-XLII-4-W2-107-2017.

Martínez-Carricondo, P., Agüera-Vega, F., Carvajal-Ramírez, F., 2020. Use of UAV-Photogrammetry for Quasi-Vertical Wall Surveying. Remote Sensing, 12(14), 2221. https://doi.org/10.3 390/rs12142221.

MAVLink Protocol Maintainers, 2020a. GCS Software implementing the MAVLink protocol. https://mavlink.io/en/about/im plementations.html (7 January 2021).

MAVLink Protocol Maintainers, 2020b. MAVLink - Micro Air Vehicle Message Marshalling Library. Communication Protocol. Dronecode. http://mavlink.io/en/ (7 January 2021).

Mostafa, M., Schwarz, K.-p., Gong, P., 1997. A Fully Digital System For Airborne Mapping. Proceedings of The International Symposium on Geodesy, Geomatics, and Navigation. http://www.navmatica.com/publication/M_Mostafa_KIS_1997. PDF.

Nesbit, P., Hugenholtz, C., 2019. Enhancing UAV-SfM 3D Model Accuracy in High-Relief Landscapes by Incorporating Oblique Images. Remote Sensing, 11(3), 239. https://doi.org/10 $.3390 /$ rs11030239.

Nex, F., Remondino, F., 2014. UAV for 3D mapping applications: a review. Applied Geomatics, 6(1), 1-15. https://doi.org/ 10.1007/s12518-013-0120-x.
Office of the Federal Register, 2020. Title 14 - Aeronautics and Space. Code of Federal Regulations, 1-1-2020 edn, 2, U.S. GOVERNMENT PUBLISHING OFFICE, Washington, DC, chapter I - Federal Aviation Administration, Department of Transportation (Continued), 925-933.

PX4 Development Team, 2020. Open Source Autopilot for Drones. PX4 Autopilot. Software. https://px4.io/ (7 January 2021).

QGroundControl Development Team, 2020. QGC - QGroundControl - Drone Control. GCS Software. http://qgroundcontrol .com/ (7 January 2021).

Rossi, P., Mancini, F., Dubbini, M., Mazzone, F., Capra, A., 2017. Combining nadir and oblique UAV imagery to reconstruct quarry topography: methodology and feasibility analysis. European Journal of Remote Sensing, 50(1), 211-221. https://doi.org/10.1080/22797254.2017.1313097.

Sammartano, G., Chiabrando, F., Spanò, A., 2020. OBLIQUE IMAGES AND DIRECT PHOTOGRAMMETRY WITH A FIXED WING PLATFORM: FIRST TEST AND RESULTS IN HIERAPOLIS OF PHRYGIA (TK). ISPRS - International Archives of the Photogrammetry, Remote Sensing and Spatial Information Sciences, XLIII-B2-2(B2), 75-82. https://www.in $\mathrm{t}$-arch-photogramm-remote-sens-spatial-inf-sci.net/XLIII-B22020/75/2020/.

senseFly, 2016. senseFly S.O.D.A. 3D - senseFly. senseFly. ht tps://www.sensefly.com/camera/sensefly-soda-3d-mappingcamera/ (7 January 2021).

Vacca, G., Dessì, A., Sacco, A., 2017. The Use of Nadir and Oblique UAV Images for Building Knowledge. ISPRS International Journal of Geo-Information, 6(12), 393. https://doi.org/ 10.3390/ijgi6120393.

Varbla, S., Puust, R., Ellmann, A., 2020. Accuracy assessment of RTK-GNSS equipped UAV conducted as-built surveys for construction site modelling. Survey Review, 0(0), 1-16. https: //doi.org/10.1080/00396265.2020.1830544.

Wu, Z., Lei, W., Sun, W., Chen, C., Wang, H., Yu, W., Zhong, H., Zhu, C., Xi, J., Yang, B., 2020. POWER TRANSMISSION LINE RECONSTRUCTION FROM SEQUENTIAL OBLIQUE UAV IMAGES. ISPRS - International Archives of the Photogrammetry, Remote Sensing and Spatial Information Sciences, XLIII-B2-2(B2), 515-520. https://doi.org/10.5194/is prs-archives-XLIII-B2-2020-515-2020.

Xie, F., Lin, Z., Gui, D., Lin, H., 2012. STUDY ON CONSTRUCTION OF 3D BUILDING BASED ON UAV IMAGES. ISPRS - International Archives of the Photogrammetry, Remote Sensing and Spatial Information Sciences, XXXIXB1(September), 469-473. https://doi.org/10.5194/isprsarchi ves-XXXIX-B1-469-2012. 